\title{
Alternative Designs of High-Temperature Superconducting Synchronous Generators
}

\author{
Kevin F. Goddard, Bartosz Lukasik, and Jan K. Sykulski, Senior Member, IEEE
}

\begin{abstract}
This paper discusses the different possible designs of both cored and coreless superconducting synchronous generators using high-temperature superconducting (HTS) tapes, with particular reference to demonstrators built at the University of Southampton using BiSCCO conductors. An overview of the electromagnetic, thermal, and mechanical issues is provided, the advantages and drawbacks of particular designs are highlighted, the need for compromises is explained, and practical solutions are offered. The scalability of results obtained from small demonstrators is considered.
\end{abstract}

Index Terms-Design methodology, finite-element method, high-temperature superconductors, magnetic analysis, superconducting devices, superconducting materials.

\section{INTRODUCTION}

$\mathbf{T}$ HIS paper is an extended version of the work originally presented at the International Conference on Electrical Machines in 2008 [1]. The text has been revised to improve clarity and focus, and more discussion has been added.

Continuing advances in the performance of high-temperature superconducting (HTS) materials open new application opportunities. HTS conductors with critical current densities as high as $200 \mathrm{~A} / \mathrm{mm}^{2}$ at $78 \mathrm{~K}$ and zero magnetic field are now available. A practical superconducting winding can easily achieve current densities of $60 \mathrm{~A} / \mathrm{mm}^{2}$ (some of the designs presented in Section VII reached $66 \mathrm{~A} / \mathrm{mm}^{2}$ ), whereas in conventional water-cooled windings, it is not realistic to use current densities in excess of $10 \mathrm{~A} / \mathrm{mm}^{2}$. The prospect of using such high-current densities to greatly increase the output power of the machine, and simultaneously reduce the losses, is very appealing [2], [3]. However, as explained in Section IV, the mechanical properties of the HTS material make it too difficult to build an HTS stator winding. In the machines considered here, superconducting coils are only used in the rotor winding.

The characteristics of the HTS materials also impose additional requirements that complicate the rotor design. The structural materials must be suitable for use at the low temperatures required by the superconductor. One of the most difficult

Manuscript received April 8, 2009; revised July 23, 2009. First published October 16, 2009; current version published November 25, 2009. This paper was recommended by Associate Editor S. W. Schwenterly. This work was supported by the Engineering and Physical Sciences Research Council (U.K.) under Grant EP/D000688/1.

K. F. Goddard and J. K. Sykulski are with the School of Electronics and Computer Science, University of Southampton, SO17 1BJ Southampton, U.K. (e-mail:kfg@ecs.soton.ac.uk; jks@soton.ac.uk).

B. Lukasik is with Areva T\&D Technology Centre, ST17 4LX Stafford, U.K. (e-mail: bartosz.lukasik@areva-td.com).

Color versions of one or more of the figures in this paper are available online at http://ieeexplore.ieee.org.

Digital Object Identifier 10.1109/TASC.2009.2031626 issues that needs to be addressed for cryogenic designs is the difference in thermal contraction between various components. To prevent this from causing high thermal stresses, it may be necessary to make the structure more flexible. However, this makes it more difficult to ensure that it is strong enough to carry the loads imposed by centrifugal forces. Another important issue is the need to limit the heat leak into the cryostat. The structure that supports the cold rotor must not conduct much heat but must have enough stiffness to keep the critical speeds out of the operating range and be able to transfer torque to the drive shaft. Extra space is also required for thermal insulation in the air gap.

To benefit from the low losses and the high current density offered by superconducting coils, the machine must be sufficiently large that these advantages are not offset by the cost of cooling and the space occupied by thermal insulation.

\section{Properties of HTS Materials}

Since the HTS materials are ceramic, they are much less tolerant to bending than conventional metallic conductors. HTS wires usually take the form of thin tapes that include a metallic matrix or substrate. This provides mechanical support to the superconductor, bridges any microcrack, and provides an alternative current path in the event that superconductivity is lost. Use of such HTS tapes restricts the shape of the coils that can be produced: flat coils are not too difficult to wind but almost any other shape would be impractical.

HTS coils can work at current densities that would make cooling virtually impossible should a conventional metal conductor be used. However, it is important to recognize that an HTS coil is not equivalent to a conventional coil with a very low resistance. The $E-J$ characteristics are highly nonlinear and greatly depend on the temperature and the strength and orientation of the magnetic field. The resistance rapidly rises with increases in temperature, current, and magnetic field with the component normal to the face of the tape having a much greater effect than the other components.

While reducing the temperature of the superconductor enables it to tolerate higher current densities and higher flux densities, it also increases the cost of cooling. Moreover, to benefit from the good heat-transfer characteristics of liquid nitrogen or liquid air, the working temperature must be restricted so that the cooling fluid does not freeze.

Although it is now possible to obtain YBCO-coated conductors in lengths and ratings suitable for the manufacture of electrical machines [4], this was not the case when the machines described here were designed; hence, BiSCCO conductors were 
selected for both demonstrators. For BiSCCO coils to carry the high current densities that are required to justify the use of a superconducting winding, at temperatures of around $65 \mathrm{~K}$, the magnetic field component normal to the face of the tape must be restricted to a relatively low value $(\approx 0.25 \mathrm{~T})$. Since the high current density in the coil can easily generate such a field, this is not easy. As previously noted, it is not practical to twist the tape to align with the field; hence, we must align the field with the tape, which requires the use of iron. In all the rotor designs considered here, as well as in an earlier transformer project [5], the field is guided by the use of permeable rings (flux diverters) to redirect much of the flux away from the superconductor. It should be noted that this would not work so well if the newer YBCO tape were used, since its field dependence is less anisotropic. Flux diverters also become less effective as the machine is scaled up, since this makes it easier to generate high flux densities that could saturate the iron and reduce their effectiveness.

\section{Choice OF Other MAterials}

While, in principle, there are a very large number of materials that could be used to construct the rotor, in reality, the choice is quite restricted; most materials were rejected either because they are expensive, or difficult to obtain, or because of the lack of relevant data to have confidence that they have suitable lowtemperature properties.

The only magnetic materials seriously considered for use at cryogenic temperatures were Invar and 9\% Ni steel. The 9\% $\mathrm{Ni}$ steel offers a higher saturation flux density and a higher thermal expansion coefficient that is a better match to the other materials considered. However, since it is not certified for use at temperatures below $73 \mathrm{~K}$, it should not be considered for use in critical structural components at such lower temperatures. In addition, although it would be cheaper in large quantities, small quantities are difficult to obtain.

The choice of nonmagnetic structural materials is, in principle, quite wide. However, in the cold parts of the rotor, we have chosen to rely on two main materials: stainless steel and G10 fiberglass, both of which are widely used in cryogenic applications; hence, considerable experience in using them and appropriate data were readily available. Alternative materials were considered only if they offered some clear advantage over each of the foregoing materials.

\section{STATOR}

It is probably impractical to build an HTS stator winding. Most common configurations require the conductors to be bent and twisted with very small radii, which would greatly reduce the critical current of the conductor. It would be difficult but possible to build the stator winding out of flat coils, but such a winding would be unlikely to make good use of space. Thus, much of the benefit of high-current densities would be lost.

Other issues that would have to be addressed if the construction of a superconducting stator were to be attempted include appropriate field shaping around the coils and the ac losses in the superconductor. Moreover, the space required for thermal insulation appears to preclude the use of a slotted stator; hence, an air-gap winding would be required, which would need to be supported by a structure that could convey the torque to the stator frame without conducting too much heat into the cryostat.

It would not be practical to apply flux diverters to the stator winding. Even if it were possible to find a shape that would make them effective, such shapes would probably be very hard to produce. The choice of the material is also a challenge. For example, the powdered iron composite used in [5] would not be effective at high flux densities, whereas solid iron rings would have high eddy-current losses that would not be acceptable in the cryostat. Even solid iron flux diverters would probably saturate if the air-gap flux density were increased above $1 \mathrm{~T}$.

The critical current of YBCO superconducting tapes is much less dependant on the normal component of the magnetic field, which suggests that they could be used without flux diverters. However, the other problems would still remain, and these appear to be prohibitive.

To reduce the cost and avoid the need to design a new stator, an existing stator is used. This was taken from a $100-\mathrm{kW}$ twopole induction machine of bore diameter $330 \mathrm{~mm}$ and iron length $325 \mathrm{~mm}$. It has 48 slots and a short-pitched (14/24) conventional two-layer star-connected winding.

\section{A. Stator Induced Rotor Losses}

The very short pitch of this winding increases the space harmonics of the stator MMF and the resulting high-frequency fields that are imposed on the rotor. The open stator slots also produce larger high-frequency fields in the rotor frame of reference. It is important that these fields should not be allowed to produce large eddy-current losses in the cold parts of the rotor, since such losses contribute to the load on the cooling system. Since the normal component of the ac field in the HTS coils can easily drive large eddy currents, it must be very low to avoid excessive ac losses. If the superconductor has sufficient spare current-carrying capacity, then it can screen itself from the normal field component, but increasing the critical current increases the loss due to the parallel field.

The cored rotor uses a cold copper screen to reduce the ac field in the HTS coils and the rotor iron. This screen encloses the assembly shown in Fig. 1 and also serves to conduct heat into the cooling pipes (see Fig. 2). With a cold conducting screen, the losses in the enclosed space are relatively unimportant, since those in the screen are substantially larger. In the coreless rotor, a warm Aluminium screen was used, which also serves as the cylindrical outer wall of the vacuum vessel. With a warm screen, the losses in the enclosed cold space must separately be considered, since they incur additional refrigeration costs. A purpose-built stator could be designed to reduce or eliminate the need for such screening.

\section{COREd Rotor Design}

The use of a magnetic rotor core greatly reduces the required rotor MMF and makes it easier to control the magnetic field in the rotor winding. This allows the rotor winding to be constructed with much less superconducting tape and to operate at a higher temperature. 


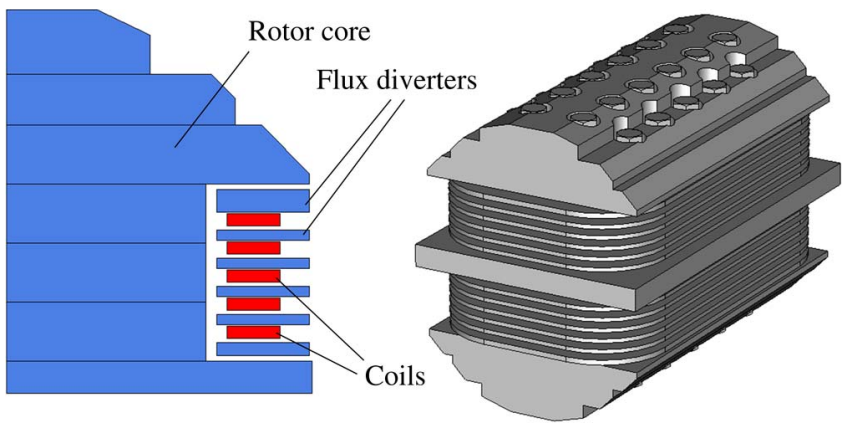

Fig. 1. Cross section of the superconducting machine with a cored rotor: (left) 2-D view and (right) 3-D view.

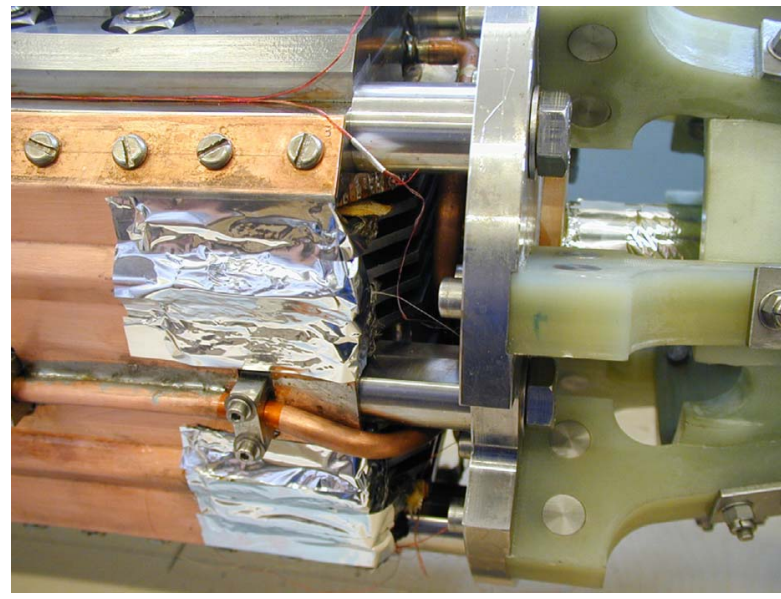

Fig. 2. Cooling pipes and copper links.

The rotor core (Fig. 1) is formed from a stack of $9 \% \mathrm{Ni}$ steel plates that are held together by $9 \% \mathrm{Ni}$ steel bolts and dowels [6]. This was considered the best material as it has good magnetic characteristics and its thermal contraction is a reasonably good match to the coils and their stainless-steel formers, thus ensuring that they do not shrink onto the pole necks. The good magnetic characteristics allow more space to be allocated to the superconducting coils and help reduce the flux density in these coils; as a result, the coils can give satisfactory performance at slightly higher temperatures. The rotor was wound with HTS tape with a nominal critical current of $120 \mathrm{~A}$ and tested with a cooling fluid temperature of $72 \mathrm{~K}$; the critical current of the rotor winding was about $93 \mathrm{~A}$, which allowed an air-gap flux density of $0.76 \mathrm{~T}$ to be obtained. Since these values were obtained from a low-speed test, they do not take into account the effects of eddy-current losses on the rotor.

\section{A. Problems With Flood Cooling}

It was originally intended that the cored design would be flood cooled. However, at the time, we could not find a satisfactory design for the mechanical connections between the rotor core and the rotating liquid vessel. The $9 \% \mathrm{Ni}$ steel used for the rotor core has a lower thermal expansion coefficient than the stainless steel proposed for the liquid vessel. To accommodate the resulting differential thermal contraction, the structure would need to be flexible; however, it must also withstand the high liquid pressure generated by centrifugal forces. While it

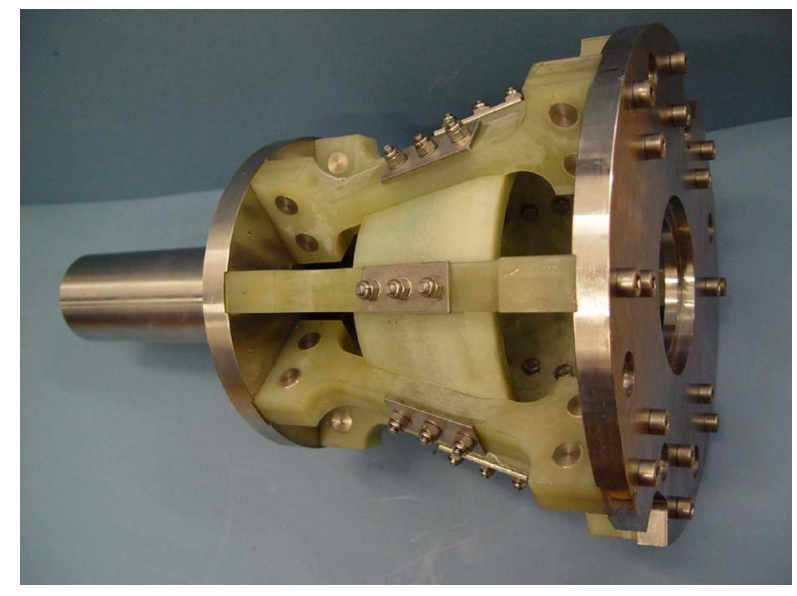

Fig. 3. Structure supporting the rotor.

cannot conclusively be proven that no satisfactory shape exists, it is clear that any solution that used these materials would be bulky. If the cylindrical wall of the liquid vessel were made from a G10 fiberglass tube, then the thermal stress would be low. However, there is a risk that the connections to the ends of this tube would leak. To avoid these difficulties, flood cooling was not used. Instead, the liquid cryogen was restricted to a few pipes with copper links employed to conduct heat into these pipes (Fig. 2).

In the course of the current project, an alternative method of connecting the inner rotor assembly to the liquid vessel was identified, which might overcome this problem; this is elaborated on in Section VI-B.

\section{B. Supporting the Cold Rotor}

The two ends of the cold rotor assembly are each supported by a conical array of G10 fiberglass beams that provide the required stiffness without conducting too much heat into the cold part of the machine (Fig. 3). The material was chosen because it has a high ratio of stiffness to thermal conductivity. One such assembly connects the cold rotor to the enclosing vacuum vessel, whereas the other is connected to a separate inner shaft that can axially slide to accommodate contraction of the cold rotor relative to the warm vacuum vessel.

\section{Coreless Rotor Design}

In principle, the use of a coreless rotor could give a significant reduction in mass of the rotor. The second project, currently underway at Southampton University, aims to build such a rotor [7]. Since the HTS tape for the cored rotor was purchased, BiSCCO tapes with substantially higher values of critical current have become available. However, there have not been substantial increases in the range of parallel and perpendicular magnetic fields over which these tapes can maintain superconductivity. This suggested that flux diverters might still be beneficial in an otherwise coreless rotor.

Removing the core introduces a set of new challenges in relation to supporting and assembling the machine, since there is no longer a rigid structure in the center. To address these 


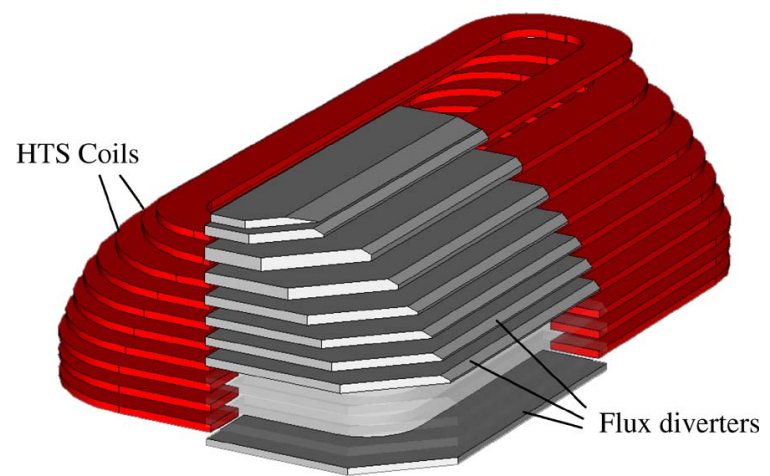

Fig. 4. First of the coreless designs showing parts of the coils and diverters (three rings are shown transparent to reveal the internal hole).

problems, a number of configurations were considered, as subsequently described. Regrettably though, none of the coreless designs considered could achieve high air-gap flux densities.

\section{A. Initial Coreless Design}

The first designs (Fig. 4) considered for the coreless rotor contained a stack of flux diverter rings interleaved with coils that are embedded in G10 support plates. Electromagnetic modeling showed that this arrangement could give a good fluxdensity waveform and acceptable flux densities in the coils. It was intended that the stack would be supported from the outside by hoop stress in the wall of the liquid vessel using two packing pieces to transfer the load. These would be made from the two halves of a G10 tube, with groves machined into the inner cylindrical face to locate the rings and coils. However, although the design looked attractive, significant problems were soon encountered, as subsequently explained.

Since the coefficient of thermal expansion normal to the layers of the G10 composite is much higher than that of the metal retaining tube, the coil formers would tend to become loose as the rotor is cooled down to its working temperature. Even without differential contraction, manufacturing tolerances would make it difficult to prevent the coils from being loose without making them tight. If they were tight, then they would tend to push the rings away from their outer supports, making it hard to transmit the acceleration torque to the outer support structure. To overcome these problems, it would be necessary to place some type of spring between each coil former and one of the adjacent rings. Since there is not enough space to put springs in these locations, the rings and coils themselves would have to act as springs. However, this would considerably increase the risk of coils getting damaged - thus lowering the reliability of the whole construction and increasing the risk that the project could not be completed.

Since the flux diverters and coil subassemblies are thin (about $6 \mathrm{~mm}$ ), they cannot be supported against centrifugal force from their outer edges alone. Additional contact forces would arise near the inner edges of the rings, which would prevent large deflections and associated stresses. However, when manufacturing tolerances are considered, it becomes clear that the balance of the rotor could change in an unpredictable manner as the speed and temperature of the cold rotor assembly are varied. Due to

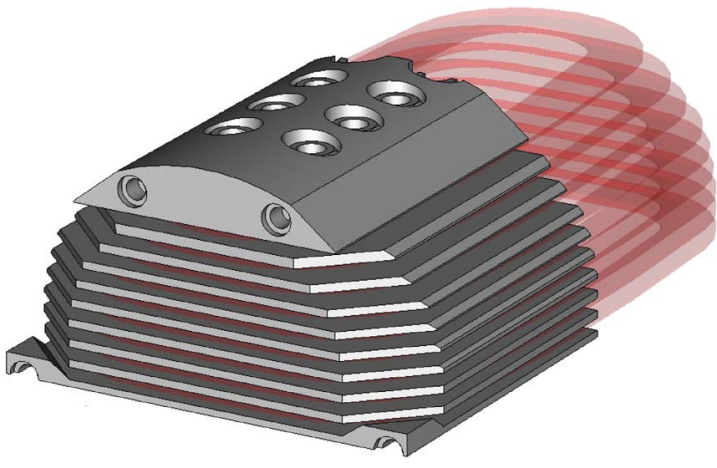

Fig. 5. Coreless design with the through bolts.

the large number of contact surfaces involved in supporting some of the rings, a large number of manufacturing tolerances contribute to this uncertainty.

Although there is plenty of space for cooling fluid in the center of the rotor, it is not easy to guide the fluid through this space. Slots or holes would have to be machined into some of the rings at their ends, and the cold fluid would have to be piped around the sides of the rotor before being allowed to flow back through the central space. The pipes would have to be placed between the two packing pieces, which would make it more difficult to center the middle coil or ring.

Having considered all these difficulties, we could not assure the reliability of the final assembly. This, together with the difficulty of machining the two packing pieces, led us to abandon this particular design.

\section{B. Alternative Coreless Design}

To overcome the problems previously described, we next considered using through bolts to hold the stack together (Fig. 5). As before, the external shape of the stack is approximately cylindrical. This maximizes the area of the coils and the air-gap flux density, which also has a reasonably good waveform; thus, magnetically, this would be a good solution. However, it proved exceedingly difficult to produce a satisfactory mechanical design.

As in the previous design, each coil would be placed on a fiberglass former extending across the central part of the machine, which allows the coils and rings to be centered by the through bolts. The use of through bolts makes the pole pieces critical structural components. It was therefore decided that they must be made from Invar. Unfortunately, this greatly increases the difficulty of adsorbing the differential thermal contraction. While finite-element modeling suggested that excessive stresses in the through bolts could be avoided by careful adjustment of clearances, this was not considered a satisfactory solution, since it would impose very tight manufacturing tolerances. A more practical solution might be to have four close-fitting bolts near the center of the rotor to align the stack, and sufficient clearance around the other bolts that thermal contraction would not create additional contacts.

Another problem arises from the differential contraction between the pole pieces and the enclosing liquid vessel. This is the same problem that led to the rejection of flood cooling in the 


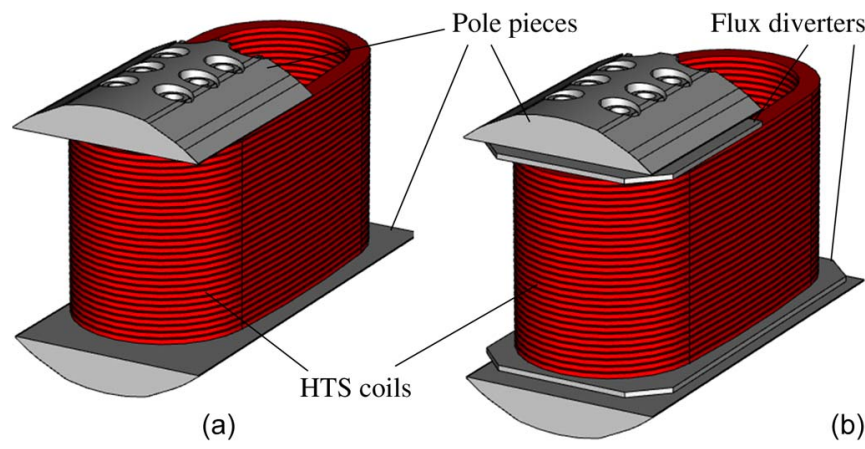

Fig. 6. Two most recent designs of the coreless rotor.

cored rotor. As previously explained, it would not be practical to make the ends of the liquid vessel sufficiently flexible to adsorb the thermal contraction while retaining sufficient strength to contain the liquid at the high pressures generated by centrifugal forces.

In the course of the current project, a possible solution to this problem was identified. In this arrangement, the ends of the liquid vessel are closed by relatively thick metal plates that can easily withstand the liquid pressure. The required flexibility is provided by a separate G10 plate that is attached to the liquid vessel near to the axis of rotation and to the inner rotor assembly at substantially greater radii. While this design would overcome the conflict between strength and flexibility, there are other requirements that need to be satisfied. In particular, the connection must reliably center the inner rotor so that the balance of the rotor does not alter too much with repeated cooling cycles and must do so with a high stiffness so that it does not significantly reduce the critical speeds. Calculations have shown that sufficient stiffness can be obtained, but full analysis to determine whether it can reliably center the rotor has not been undertaken. There is also some concern that the G10 could be damaged by contact pressure or by sliding during cooling or warming.

The entire cold part would be supported within the vacuum vessel by a similar structure to that described in Section V-B.

While it might be possible to produce a satisfactory mechanical design, there was insufficient confidence to justify further work.

\section{Final Coreless Design}

In this design, all the coils are of the same size and are mounted on a thin-walled tube in the middle of the rotor (Fig. 6). With this arrangement, the perpendicular component of the magnetic field of a coil is largely counteracted by the magnetic field produced by nearby coils. It is therefore possible to remove the $9 \% \mathrm{Ni}$ plates from between the coils. However, since the coils are no longer shielded from the stator winding field, the current in each coil has to be reduced to ensure low $\mathrm{E}$ fields in the superconductor. Retaining the pole pieces reduces the perpendicular field in the last few coils, where the highest values occur, and allows for some shaping of the air gap flux density waveform, which is still worse than that in the previous coreless designs. Due to a lower current and a smaller coil area, the air gap flux density is also lower than in the previous design.
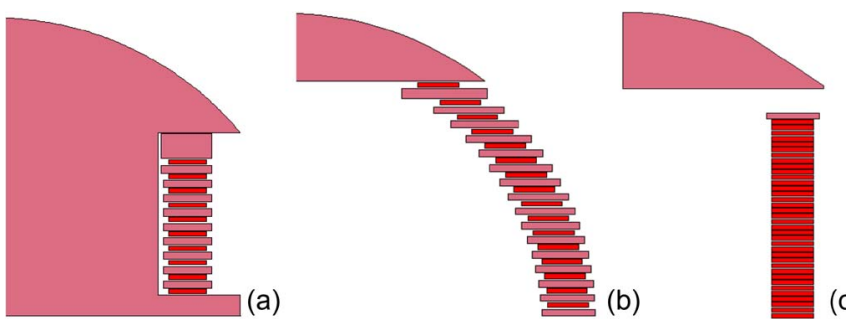

Fig. 7. Three scaled-up designs analyzed, showing only the HTS coils and ferromagnetic components of the rotor.

To minimize the cold mass that must be supported with minimum heat leak, the pole pieces are warm and placed outside the cryostat. Since displacing the pole pieces away from the rotor winding causes the perpendicular magnetic field in the last few coils to increase, it was necessary to replace the flux diverters at the top and bottom of the winding [Fig. 6(b)]. In this design, only the coils and the two flux diverters are enclosed in the stainless-steel vessel flooded with liquid cryogen. This stainless steel vessel consists of two concentric thin-walled oval tubes with thin reinforcing ribs and two thicker rings that are welded to the tubes to close the vessel. The inner tube supports the coils and allows the heat through bolts to pass through, whereas the rings also provide attachment points for the structures that support the cold rotor assembly. Despite the somewhat inferior electromagnetic performance, the significant advantages in lower mass, simpler assembly, higher reliability, easier and cheaper manufacturing of the coils (as they are all identical), and overall simplicity have proven deciding factors in selecting this design over those discussed earlier. It should not be taken for granted, however, that one of the suggested alternatives would not offer a competitive solution-should an answer to the mechanical and thermal problems highlighted be found.

\section{SCAlability of the Proposed Designs}

As previously noted, an HTS rotor can be beneficial only if the high current density and the low loss of the winding are not offset by the space required for thermal insulation and the power required by the cooling system. For a coreless rotor to be beneficial, it is also necessary that the reduction in rotor mass is not offset by an increase in the mass of the stator. Both the cored machine already built and the coreless version now being constructed at the University of Southampton are too small to satisfy these conditions. We therefore decided to investigate the possibility of scaling up three of the described designs. Fig. 7 shows the designs considered. The first design is similar to that described in Section V, whereas the other two are similar to those described in Sections VI-B and C. These designs were sized to fit a stator with a bore diameter of $660 \mathrm{~mm}$ - which is twice the size of that used for testing at Southampton.

As a first step, 2-D electromagnetic models were used to determine the maximum air-gap flux density that could be achieved with each of the rotor designs. The air-gap flux density is important since a low value would increase the mass and losses of the stator, which might easily offset the weight-saving 


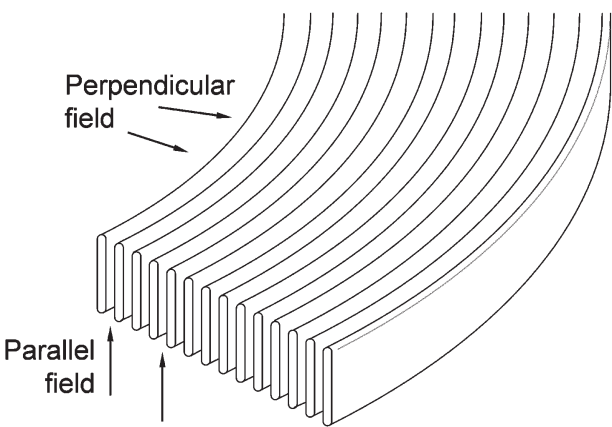

Fig. 8. Field component orientation relative to HTS coils.

advantage of a coreless rotor and possibly the energy-saving advantage of a superconducting rotor as well.

Since manufacturer's graphs showing the critical current of HTS tape as a function of the magnetic flux density are usually available only for fields normal or parallel to the face of the tape, some form of interpolation is required to estimate the critical current when the field is at some intermediate angle. To do this, an equivalent magnetic flux density $\left(B^{\prime}\right)$ was defined as a function of the parallel $\left(B_{\|}\right)$and perpendicular $\left(B_{\perp}\right)$ components of the applied field and a scaling factor $(k)$ as

$$
B^{\prime}=\sqrt{B_{\perp}^{2}+\left(\frac{B_{\|}}{k}\right)^{2}} .
$$

Fig. 8 shows the orientation of these field components relative to the HTS coils. For the BiSCCO tapes considered here, it was found that a single value of the scaling factor allowed both sets of manufacturer's graphs to be fitted by graphs of critical current as a function of $B^{\prime}$.

For each of the configurations shown in Fig. 7, models were built with various numbers of turns in the rotor coils. Each of these models was solved for a number of values of field current and postprocessed to find the maximum value of $B^{\prime}$ in the coils. By fitting curves to the graphs of $B^{\prime}$ as a function of current and of critical current as a function of $B^{\prime}$, the current was found such that the minimum value of the critical current in the coils is equal to the imposed current. This value was taken to be the critical current of the whole winding. Since it was assumed that interest lies in building the machine cooled by liquid nitrogen or liquid air, the critical current was estimated for winding temperatures of 70,66 , and $60 \mathrm{~K}$. Cooling to 70 or $66 \mathrm{~K}$ can be achieved using liquid nitrogen, whereas liquid air would also permit cooling to $60 \mathrm{~K}$.

The predicted critical current at $66 \mathrm{~K}$ has been plotted as a function of the number of turns in the coils, together with the resulting air-gap flux density. Fig. 9 shows these graphs for each of the three rotor configurations considered. The values of critical current shown in these graphs are probably high since they are obtained from no-load models, which do not include the effect of the load current on the field in the coils.

The cored design produces the highest values of air-gap flux density and uses the least superconducting tape, since the core greatly reduces the magnetizing current. The graphs for this design show that an air-gap flux density exceeding $1 \mathrm{~T}$ can be obtained with 80-turn coils carrying $140 \mathrm{~A}$. While this current

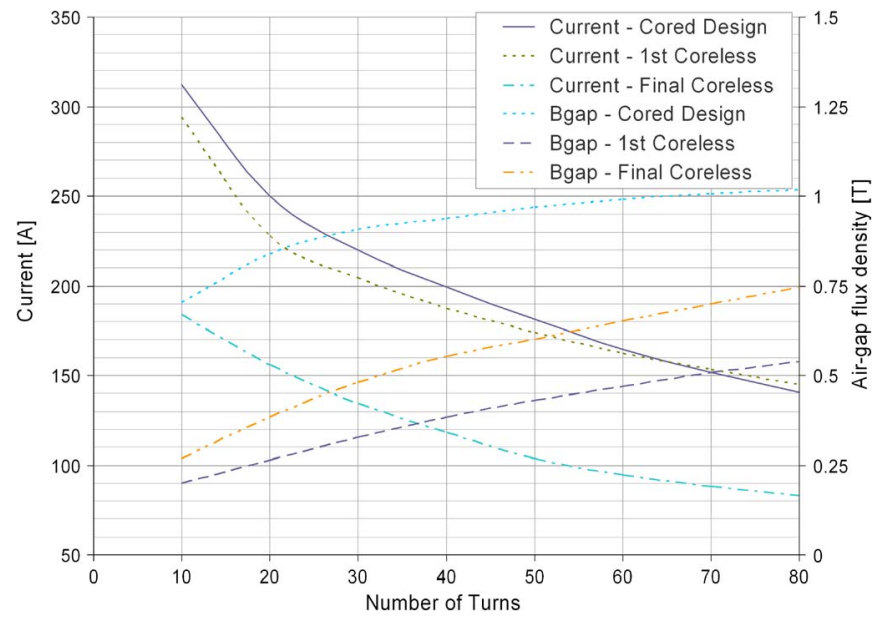

Fig. 9. Current and air-gap flux density as functions of the number of turns for various designs at $66 \mathrm{~K}$.

may be a little high, and the end effects will tend to reduce the air-gap flux density produced by any given current, it does show that a cored HTS rotor of this size can make efficient use of the stator. However, increasing the size of the rotor makes many of the mechanical difficulties considerably worse: stresses due to centrifugal force tend to increase in proportion to the square of the rotor diameter; the cross-sectional area of the beams that support the cold rotor assembly would need to increase at least in proportion to rotor mass to avoid significant reductions in the critical speeds of the rotor. The achievable air-gap flux density rises from $0.975 \mathrm{~T}$ at $70 \mathrm{~K}$ to $1.1 \mathrm{~T}$ at $60 \mathrm{~K}$; this small increase and the shape of the curve in Fig. 9 both suggest that magnetic saturation is the limiting factor.

Since the two coreless designs do not achieve sufficiently high values of air-gap flux density to saturate the stator core and have no rotor core that could saturate, the air-gap flux density is mainly determined by two factors: 1) the total ampere turns in the rotor winding and 2) the mean area of these turns. The graphs show that the first coreless design gives higher values of critical current; this is because the increased use of flux diverters reduces $B_{\perp}$ in the coils. It is also apparent that the critical current reduces as the number of turns in the rotor coils and the air-gap flux density are increased. This occurs because it becomes increasingly difficult to control the magnetic field in the topmost coils. The top diverters and the tips of the pole piece tend to saturate because they are in the region where the perpendicular component of the magnetic field driven by the coils is highest. Flux attracted to the stator core also makes a significant contribution to $B_{\perp}$ in this region.

The first coreless design can give an air-gap flux density of nearly $0.55 \mathrm{~T}$ at $66 \mathrm{~K}$, which increases to above $0.67 \mathrm{~T}$ at $60 \mathrm{~K}$. In comparison, it can be seen that the final coreless design can achieve an air-gap flux density of $0.75 \mathrm{~T}$ at $66 \mathrm{~K}$ using 80 -turn coils carrying $83 \mathrm{~A}$. Moreover, by cooling to $60 \mathrm{~K}$, a value of nearly $1 \mathrm{~T}$ can be achieved. While such high values of air-gap flux density would allow efficient use of the stator, the final design does not make efficient use of the superconducting tape; it contains twice as much HTS tape as the other coreless designs. 


\section{CONCLUSION}

This paper has discussed practical considerations relating to possible designs of synchronous generators built with HTS tapes in the rotor and has explained the engineering compromises between electromagnetic, thermal, and mechanical requirements that need to be made. Both cored and coreless designs have been explored and found to be possible. A cored rotor was built and tested, whereas several scenarios have been considered for the coreless version, and one is being pursued (expected to be completed in 2009). Both demonstrators have used BiSCCO tapes, but the potential impact of the commercial availability of the new $2 \mathrm{G}$ YBCO conductors has briefly been addressed. Finally, a way to scale up the results from small demonstrators to full-size machines has been proposed, but it is recognized that scalability is a challenging issue with somewhat limited applicability.

\section{REFERENCES}

[1] K. F. Goddard, B. Lukasik, and J. K. Sykulski, "Alternative designs of a superconducting synchronous generator: The Southampton approach," presented at the Int. Conf. Electrical Machines, Vilamoura, Portugal, Sep., 2008, Paper 1485.

[2] P. N. Barnes, M. D. Sumption, and G. L. Rhoads, "Review of high power density superconducting generators: Present state and prospects for incorporating YBCO windings," Cryogenics, vol. 45, no. 10/11, pp. 670-686, 2005.

[3] S. S. Kalsi, K. Weeber, H. Takesue, C. Lewis, H. W. Neumueller, and R. D. Blaugher, "Development status of rotating machines employing superconducting field windings," Proc. IEEE, vol. 92, no. 10, pp. 16881704, Oct. 2004.

[4] A. P. Malozemoff, S. Fleshler, M. Rupich, C. Thieme, X. Li, W. Zhang, A. Otto, J. Maguire, D. Folts, J. Yuan, H.-P. Kraemer, W. Schmidt, M. Wohlfart, and H.-W. Neumueller, "Progress in high temperature superconductor coated conductors and their applications," Supercond. Sci. Technol., vol. 21, no. 3, p. $034005,2008$.

[5] J. K. Sykulski, K. F. Goddard, and R. L. Stoll, "High temperature superconducting demonstrator transformer: Design considerations and first test results," IEEE Trans. Magn., vol. 35, no. 5, pp. 3559-3561, Sep. 1999.
[6] M. K. Al-Mosawi, C. Beduz, and Y. Yang, "Construction of a 100 kVA high temperature super-conducting synchronous generator," IEEE Trans. Appl. Supercond., vol. 15, no. 2, pp. 2182-2185, Jun. 2005.

[7] M. K. Al-Mosawi, K. Goddard, C. Beduz, and Y. Yang, "Coreless HTS synchronous generator operating at liquid nitrogen temperatures," IEEE Trans. Appl. Supercond., vol. 17, no. 2, pp. 1599-1602, Jun. 2007.

Kevin F. Goddard is currently a Research Fellow in electrical power engineering in the School of Electronics and Computer Science, University of Southampton, Southampton, U.K. His research interests include the calculation of electromagnetic fields, application of numerical modeling to the design and optimization of electrical machines, and application of high-temperature superconductors.

Bartosz Lukasik is currently a Research Technologist at the Areva T\&D Technology Centre, Stafford, U.K. His research interests include applied superconductivity, computational electromagnetics, and optimization.

Jan K. Sykulski (M'92-SM'94) is Professor of Applied Electromagnetics and Head of Electrical Power Engineering, School of Electronics and Computer Science, University of Southampton, Southampton, U.K. His personal research is in development of fundamental methods of computational electromagnetics, power applications of high-temperature superconductivity, simulation of coupled field systems, and design and optimization of electromechanical devices. He has published nearly 300 scientific papers. His personal web page, which includes all his publications, is http://www.ecs.soton.ac.uk/info/people/jks.

Prof. Sykulski is Past Chair of the Professional Network Electromagnetics of the Institution of Engineering and Technology, U.K., founding Secretary of the International Compumag Society, a Visiting Professor at universities in Canada, France, Italy, Poland and China, Editor-in-Chief of COMPEL (Emerald) and a member of the International Steering Committees of several international conferences. He is a Fellow of the Institution of Engineering and Technology, U.K., a Fellow of the Institute of Physics, a Fellow of the British Computer Society, and has an honorary title of Professor awarded by the President of Poland. 